BEAGLES, A. E.; SÄNDIG, A.-M.

\title{
Singularities of Rotationally Symmetric Solutions of Boundary Value Problems for the Lamé Equations
}

\begin{abstract}
Wir wenden die Theorie elliptischer Randwertprobleme in nichtglatten Gebieten mit konischen Punkten auf rotationssymmetrische Lösungen von Randwertproblemen für Lamé-Gleichungen an. Die resultierende Entwicklung zieht singuläre Vektor-Funktionen nach sich, die dann wieder von einem Parameter $\alpha$ abhängen. Wir stellen hier Gleichungen vor, die die Werte von $\alpha$ entweder für unbelastete oder für Dirichletsche Rand-Bedingungen bestimmen. Wir geben einen numerischen Algorithmus an, mit dem $\alpha$ berechnet werden kann und bringen einige Grafiken für die erhaltenen Werte. Die singulären Vektor-Funktionen werden explizit gegeben, und wir stellen Gleichungen zur Berechnung der Entwicklungskoeffizienten vor.
\end{abstract}

We apply the theory of elliptic boundary value problems in non-smooth domains with conical points to rotationally symmetric solutions of boundary value problems for the Lamé equations. The resulting expansion involves singular vector-functions which, in turn, depend on a parameter, $\alpha$. We here present equations which determine the values of $\alpha$ for either stress-free or Dirichlet boundary conditions. We give a numerical algorithm whereby $\alpha$ can be computed and present some plots of the values obtained. The singular vector-functions are given explicitly and we present equations for the computation of the coefficients of the expansion.

Применяем теорию эллитических краевых задач в негладких областях с коническими точками на врачательносимметрические решения краевых задач дяя уравнений Ламе. Проистекаючее разложнение вовлекает сингулярные векторные функиии, которые, по очереди, зависят от параметра $\alpha$. Здесь представляем уравнения определяюииеся значения от $\alpha$ или для ненапряженных или для краевых условий Дирихле. Даем численный алгоритм, где можно вычислить $\alpha$ и представляем несколько графиков полученных значений. Сингулярные векторные функции даются в явном форме и представляются уравнения для вычисления коэффиииентов разложения.

MSC (1980): $35 J 55$

\section{Introduction}

It is now well known that singularities are present in the solution of the Lamé equations in conical domains. The general theory, with two-dimensional applications is available in reference [1] and the Lamé equations are considered in reference [2]. From this theory it is known that the radial $(r)$ dependence of singular vector-functions is of the form $r^{\alpha} \ln ^{q}{ }^{q} r$ where $q$ is an integer.

Here we are concerned with rotationally symmetric solutions of the Lamé equations with prescribed displacements or stresses near a circular conical boundary point. This application of the theory of elliptic boundary value problems in non-smooth domains with conical points is, to our knowledge, completely original, though some values of $\alpha$ for $q=0$ in the context of stress-free or zero displacement boundary conditions have been computed, see reference [3].

In this paper we formulate the regularity problem in weighted Sobolev spaces, thus motivating the calculation of $\alpha$. We consider bounds on the values of $q$ and give a complete description of the singular vector-functions that appear in the asymptotic expansion near the conical point. This involves the solution of transcendental equations involving Legendre functions. We describe an efficient method for the computation of these functions and solving the equations, and include some plots of the resulting solutions. Finally, we present the singular vector-function expansion and give equations whereby the coefficients appearing in the expansion can be calculated.

\section{Formulation of the problem}

Let $\Omega$ be a three-dimensional bounded domain with a circular conical point $\theta$ on its boundary (see Fig. 1). Assume that the displacement field $\boldsymbol{u}(\boldsymbol{x})$ of this isotropic elastic body satisfies the linear equation system

or

$$
\begin{aligned}
& \boldsymbol{L} u \equiv \boldsymbol{L}\left(D_{x}\right) \boldsymbol{u}(\boldsymbol{x}) \equiv \mu \Delta u(x)+(\lambda+\mu) \nabla(\nabla \cdot u(x))=-f(x) \text { for } x \in \Omega, \\
& \boldsymbol{L}_{1} u \equiv \boldsymbol{L}_{1}\left(D_{x}\right) \boldsymbol{u}(\boldsymbol{x}) \equiv \boldsymbol{u}(\boldsymbol{x})=g_{1}(x) \text { for } x \in \partial \Omega,
\end{aligned}
$$

$$
L_{2} u \equiv L_{2}\left(D_{x}\right) u(x) \equiv S(u(x)) \cdot M(x)=g_{2}(x) \text { for } x \in \partial \Omega,
$$

where $\lambda$ and $\mu$ are the Lamé constants, $\boldsymbol{f}(\boldsymbol{x})$ is the vector of the volume forces, $\boldsymbol{g}_{1}(\boldsymbol{x})$ is a prescribed displacement (Dirichlet conditions), $\boldsymbol{g}_{2}(\boldsymbol{x})$ is a traction (Neumann conditions). $S(\boldsymbol{u}(\boldsymbol{x}))$ denotes the stress tensor, with Cartesian components

$$
\left[S(\boldsymbol{u}(\boldsymbol{x})]_{i j} \equiv \mu\left[\frac{\partial u_{i}}{\partial x_{j}}+\frac{\partial u_{j}}{\partial x_{i}}\right]+\delta_{i j} \lambda \nabla \cdot \boldsymbol{u}(\boldsymbol{x}),\right.
$$

where $u_{i}$ is the $i$-th component of $\boldsymbol{u}(\boldsymbol{x})$ and $\delta_{i j}$ is the Kronecker symbol. $\boldsymbol{M}(\boldsymbol{x})$ is the unit vector of the outward normal to $\partial \Omega$ at the point $x$. 


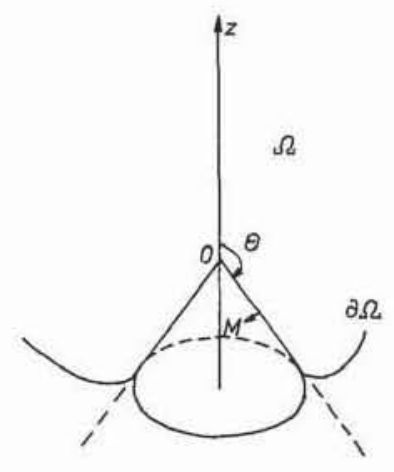

Fig. 1. A body with a rotationally symmetric conical vertex

We are interested in the behaviour of the solution $\boldsymbol{u}$ of problem (2.1), (2.2) and (2.1), (2.3) near the apex 0 . This is a local problem. Therefore we introduce spherical coordinates $(r, \theta, \varphi)$ and multiply $\boldsymbol{u}(\boldsymbol{x})$ by a cut-off function $\eta=\eta(|x|)=\eta(r) \in C^{\infty}(0, \infty)$, where $0 \leqq \eta(r) \leqq 1$ and

$$
n(r) \equiv \begin{cases}1 & \text { for } 0 \leqq r \leqq \delta \\ 0 & \text { for } r \geqq 2 \delta .\end{cases}
$$

The number $\delta$ is so small that the ball $B_{2 \delta}(\theta)$ intersected with $\Omega$ coincides with the cone

$$
K_{\delta} \equiv\left\{(r, \theta, \varphi): 0<r<\delta, 0 \leqq \theta<\theta_{0}, 0 \leqq \varphi<2 \pi\right\} .
$$

Let $\boldsymbol{w}=\eta \boldsymbol{u}$. The field $\boldsymbol{w}$ satisfies the following boundary value problem in the infinite cone $K \equiv K_{\infty}$,

$$
\begin{aligned}
& L w=F(x, u(x)) \text { for } x \in K, \\
& L_{i} w=G_{i}(x, u(x)) \text { for } x \in \partial K, \quad i=1 \text { or } 2,
\end{aligned}
$$

where the right hand sides $\boldsymbol{F}, \boldsymbol{G}_{\boldsymbol{i}}$ can be computed from $\boldsymbol{f}, \boldsymbol{g}_{\boldsymbol{i}}, \eta$ and the unknown field $\boldsymbol{u}$ and its first derivatives.

The analysis of the solvability and regularity of problem (2.6), (2.7) is well developed in the framework of standard Sobolev spaces [4], [5] or in the framework of weighted Sobolev spaces $[1,2,6,7]$.

We consider the theory in weighted Sobolev spaces and define:

$$
V^{k, p}(K, \beta)
$$

to be the closure of the set

$$
C_{M}^{\infty}(K) \equiv\left\{v \in C^{\infty}(\bar{K}) \text {, supp } v \text { bounded, supp } v \cap M=\emptyset \text { for } M=\{\boldsymbol{\theta}\}\right\}
$$

with respect to the norm

$$
\left\|v ; V^{k, p}(K, \beta)\right\|=\left(\sum_{|\gamma| \leqq k} \int_{K}\left|D^{\gamma} v(x)\right|^{p} r^{p(\beta-k+|\gamma|)} \mathrm{d} x\right)^{1 / p},
$$

where we use standard multi-index notation. We also let

$$
V^{k-1 / p, p}(\partial K, \beta)
$$

be the space of traces, defined as the factor space

$$
V^{k, p}(K, \beta) / V^{k, p}(K, \beta, \partial K),
$$

where $V^{k, p}(K, \beta, \partial K)$ is the closure of $C_{\partial k}^{\infty}(K)$ with respect to the norm (2.9). The weighted space $V^{k, p}(\Omega, \beta)$ is defined analogously to $V^{k, p}(K, \beta)$.

We now consider the operators corresponding to the problem (2.6), (2.7)

$$
\begin{aligned}
A_{i}\left(D_{x}\right) \equiv & \left\{L\left(D_{x}\right), L_{i}\left(D_{x}\right)\right\}:\left[V^{l+2, p}(K, \beta)\right]^{3} \\
& \rightarrow\left[V^{l, p}(K, \beta)\right]^{3} \times\left[V^{l+3-i-1 / p, p}(\partial K, \beta)\right]^{3}, \quad i=1 \text { or } 2,
\end{aligned}
$$

where $[X]^{3}=X \times X \times X$.

Introducing in (2.6), (2.7) polar coordinates $(r, \theta, \varphi)$ and using the Mellin transform with respect to $r$,

$$
\hat{w}(\alpha, \theta, \varphi)=\frac{1}{\sqrt{2 \pi}} \int_{0}^{\infty} r^{-\alpha-1} w(r, \theta, \varphi) \mathrm{d} r,
$$

we get for $l=0, p=2$ a parameter dependent boundary value problem with the operators

$$
A_{i}\left(\alpha, D_{\omega}\right)=\left\{L\left(\alpha, D_{\omega}\right), L_{i}\left(\alpha, D_{\omega}\right)\right\}:\left[W^{2,2}(S)\right]^{3} \rightarrow\left[L^{2}(S)\right]^{3} \times\left[W^{21 / 2-i, 2}(\partial S)\right]^{3},
$$

where $\omega=(\theta, \varphi), S$ is the solid angle of the cone $K$ and $W^{k, p}(S)$ are the classical Sobolev spaces. 
The distribution of the eigenvalues of the operators $A_{i}\left(\alpha, D_{\omega}\right)$ determines the regularity and solvability of the original problem (2.1), (2.2) and (2.1), (2.3).

Definition 1: The complex number $\alpha=\alpha_{n}$ is an eigenvalue of $\boldsymbol{A}_{i}\left(\alpha, D_{\omega}\right), i=1$ or 2 , if there exist $I_{n}$ ( $\left.\geqq 1\right)$ nontrivial linearly independent solutions $e_{\sigma}^{0}\left(\alpha_{n}, \omega\right)$ in $\left[W^{2,2}(S)\right]^{3}, \sigma=1, \ldots, I_{n}$, of the system $A_{i}\left(\alpha, D_{\omega}\right) e(\alpha, \omega)=0$ for $\alpha=\alpha_{n}$. $e_{\sigma}^{0}\left(\alpha_{n}, \omega\right)$ is an eigenvector-function of $A_{i}\left(\alpha, D_{\omega}\right)$ with respect to $\alpha_{n}$. The vector functions $e_{\sigma}^{1}\left(\alpha_{n}, \omega\right), \ldots, e_{\sigma}^{k-1}\left(\alpha_{n}, \omega\right)$ are associate vector-functions to $\alpha_{n}$ and $e_{\sigma}^{0}\left(\alpha_{n}, \omega\right)$ if

$$
\sum_{j=0}^{s} \frac{1}{j !} \frac{\partial^{j} A_{i}\left(\alpha_{n}, D_{\omega}\right)}{\partial \alpha^{j}} e_{\sigma}^{s-j}\left(\alpha_{n}, \omega\right)=0
$$

for $s=0, \ldots, k-1$. The vector-functions $\boldsymbol{e}_{\sigma}^{0}, \ldots, \boldsymbol{e}_{\sigma}^{k-1}$ generate a Jordan chain of length $k$.

The following solvability and regularity theorems are formulated in references [1], [6], [7] and [8].

Theorem 1 (Solvability): The operators (2.10) are isomorphisms iff no eigenvalue of $A_{i}\left(\alpha, D_{\omega}\right)$ lies on the line $\operatorname{Re}(\alpha)=-\beta-3 / p+2+l$.

Theorem 2 (Regularity): Assume that the right-hand-sides of (2.6) and (2.7), $\boldsymbol{F}$ and $\boldsymbol{G}_{\boldsymbol{i}}$ satisfy

$$
\boldsymbol{F} \in\left[V^{l, p}(K, \beta)\right]^{3} \cap\left[V^{l^{\prime}, p^{\prime}}\left(K, \beta^{\prime}\right)\right]^{3}
$$

and

$$
\boldsymbol{G}_{i} \in\left[V^{l+3-i-1 / p, p}(\partial K, \beta)\right]^{3} \cap\left[V^{l^{\prime}+3-i-1 / p^{\prime}, p^{\prime}}\left(\partial K, \beta^{\prime}\right)\right]^{3} .
$$

If no eigenvalues of $A_{i}\left(\alpha, D_{\omega}\right)$ lie on the lines

$$
\operatorname{Re}(\alpha)=-\beta-\frac{3}{p}+l+2 \equiv-h \text { and } \operatorname{Re}(\alpha)=-h^{\prime} \equiv-\beta^{\prime}-\frac{3}{p^{\prime}}+l^{\prime}+2
$$

and if the eigenvalues $\alpha_{1}, \alpha_{2}, \ldots, \alpha_{N}$ are situated in the strip $-h<\operatorname{Re}(\alpha)<-h^{\prime}$, then the solution of (2.6), (2.7), $\boldsymbol{w} \in\left[V^{l+2, p}(K, \beta)\right]^{3}$ allows the following expansion,

$$
w(r, \omega)=\sum_{n=1}^{N} \sum_{\sigma=1}^{I_{n}} \sum_{k=0}^{J_{\sigma n}-1} C_{\sigma k n} \omega_{k, n}^{\sigma}(r, \omega)+v(r, \omega),
$$

where $v(r, \omega) \in\left[V^{l^{\prime}+2, p^{\prime}}\left(K, \beta^{\prime}\right)\right]^{3}$,

$$
I_{n} \equiv \operatorname{dim} \operatorname{ker}\left(A_{i}\left(\alpha, D_{\omega}\right)=\operatorname{dim} \operatorname{span}\left\{e_{1}^{0}\left(\alpha_{n}, \omega\right), \ldots, e_{I_{n}}^{0}\left(\alpha_{n}, \omega\right)\right\}\right.
$$

is the number of linearly independent eigenvector-functions to $\alpha_{n} . J_{o n}$ is the length of the Jordan chains, $C_{\sigma k n}$ are constants and

$$
\boldsymbol{w}_{k, n}^{\sigma}(r, \omega)=r^{\alpha_{n}} \sum_{s=0}^{k}(\ln r)^{s} e_{\sigma}^{k-s}\left(\alpha_{n}, \omega\right)
$$

are the so-called singular vector-functions.

We now go back to our problems (2.1), (2.2) and (2.1), (2.3), assuming that

$$
\boldsymbol{w} \equiv \eta \boldsymbol{u} \in\left[V^{l+2, p}(K, \beta)\right]^{3}, \quad \eta \boldsymbol{f} \in\left[V^{l, p}(K, \beta)\right]^{3} \cap\left[V^{l^{\prime}, p^{\prime}}\left(K, \beta^{\prime}\right)\right]^{3}
$$

and

$$
\eta g_{i} \in\left[V^{l+3-i-1 / p, p}(\partial K, \beta)\right]^{3} \cap\left[V^{l^{\prime}+3-i-1 / p^{\prime}, p^{\prime}}\left(\partial K, \beta^{\prime}\right)\right]^{3}, \quad i=1,2 .
$$

Then the right hand sides of (2.6) and (2.7) satisfy the suppositions of theorem 2 and we have

Theorem 3: Let $\boldsymbol{u}$ be a solution of problem (2.1), (2.2) or (2.1), (2.3) for which the above suppositions are satisfied. Then the expansion

$$
\boldsymbol{u}(r, \omega)=\sum_{n=1}^{N} \sum_{\sigma=1}^{I_{n}} \sum_{k=0}^{J_{\sigma n}-1} \eta C_{\sigma k n} w_{k, n}^{\sigma}(r, \omega)+u_{0}(r, \omega)
$$

holds, where $u_{0} \in\left[V^{l^{\prime}+2, p^{\prime}}\left(\Omega, \beta^{\prime}\right)\right]^{3}$ and $-h<\operatorname{Re}\left(\alpha_{n}\right)<-h^{\prime}, n=1, \ldots, N$.

Proof: Using expansion (2.13) we get for

$$
\begin{aligned}
\boldsymbol{u}(r, \omega) & =\eta^{2} \boldsymbol{u}(r, \omega)+\left(1-\eta^{2}\right) \boldsymbol{u}(r, \omega)=\eta \boldsymbol{w}(r, \omega)+\left(1-\eta^{2}\right) \boldsymbol{u}(r, \omega) \\
& =\sum_{n=1}^{N} \sum_{\sigma=1}^{I_{n}} \sum_{k=0}^{J_{\sigma n}-1} C_{\sigma k n} \eta \boldsymbol{w}_{k, n}^{\sigma}(r, \omega)+\eta \boldsymbol{v}(r, \omega)+\left(1-\eta^{2}\right) \boldsymbol{u}(r, \omega) .
\end{aligned}
$$

Setting $u_{0}=\eta v(r, \omega)+\left(1-\eta^{2}\right) \boldsymbol{u}(r, \omega)$ we get $(2.15)$. 
Remark: The assumption $\left.\boldsymbol{w}=\eta \boldsymbol{u} \in V^{l+2, p}(K, \beta)\right]^{3}$ is always satisfied for an appropriate $\beta$. If we start from a weak solution $\boldsymbol{u} \in\left[\mathscr{W}^{1.2}(\Omega)\right]^{3}$ to $(2.1),(2.2)$ for the Dirichlet problem with homogeneous Dirichlet conditions (i.e. $\left.\boldsymbol{g}_{1}(\boldsymbol{x}) \equiv \boldsymbol{0}\right)$ then $u$ is contained in

$$
\left[V^{2,2}(\Omega, 1)\right]^{3} \text { or even in }\left[V^{2,2}\left(\Omega,-\operatorname{Re}\left(\alpha_{0}\right)+\frac{1}{2}+\varepsilon\right)\right]^{3},
$$

where $\varepsilon>0$ is a small real number, $\alpha_{0}$ is the eigenvalue with smallest (positive) real part and $f$ is sufficiently smooth.

If we consider the weak solution $\boldsymbol{u} \in\left[W^{1,2}(\Omega)\right]^{3}$ of the Neumann problem (i.e. $\left.g_{2}(x) \equiv \boldsymbol{\theta}\right)$ we get that $\boldsymbol{u}$ is also from $\left[V^{2,2}\left(\Omega,-\operatorname{Re}\left(\alpha_{0}\right)+\frac{1}{2}+\varepsilon\right)\right]^{3}$ for an appropriate $f$. Therefore we have to consider the eigenvalues $\alpha$ with $\operatorname{Re}(\alpha)>-\frac{1}{2}$ starting from $u \in\left[V^{2,2}(\Omega, 1)\right]^{3}$. We will see later that the strip $-\frac{1}{2} \leqq \operatorname{Re}(\alpha)<0$ is free of eigenvalues.

Now we are able to formulate our problem in the context of weighted Sobolov spaces:

Calculate the singular part of expansion (2.15), i.e. calculate $I_{n}, J_{\sigma n}, C_{\sigma k n}$ and $\boldsymbol{w}_{k n}^{\sigma}(r, \omega)$.

\section{Rotationally symmetric solutions of the Lamé equations}

When studying problems over bodies with circular conical points (such as that shown in Fig. 1) it is natural to use spherical polar coordinates $(r, \theta, \varphi)$ with origin at the apex $\boldsymbol{\theta}$. In these coordinates the local orthonormal basis vectors are

$$
\begin{aligned}
& e_{r} \equiv(\sin \theta \cos \varphi, \sin \theta \sin \varphi, \cos \theta)^{\top}, \quad e_{\theta} \equiv(\cos \theta \cos \varphi, \cos \theta \sin \varphi,-\sin \theta)^{\top}, \\
& e_{\varphi} \equiv(-\sin \varphi, \cos \varphi, 0)^{\top},
\end{aligned}
$$

so that any vector $u$ can be written as $u \equiv u_{\mathrm{r}} e_{r}+u_{\theta} e_{\theta}+u_{\varphi} e_{\varphi}$. Any rotationally symmetric vector field will have the form

$$
\boldsymbol{u}(\boldsymbol{x})=u_{r}(r, \theta) \boldsymbol{e}_{r}+u_{\theta}(r, \theta) \boldsymbol{e}_{\theta} \equiv\left(e_{r} e_{\theta}\right) \tilde{\boldsymbol{u}}(r, \theta) .
$$

The displacement field in a rotationally symmetric body will have this form if all the forces acting on it are themselves rotationally symmetric. We seek singular vector-functions (see (2.14)) of the form

$$
\boldsymbol{w}_{0}^{\sigma}(r, \omega) \equiv r^{\alpha}\left(G_{r}(\theta) e_{r}+G_{\theta}(\theta) e_{\theta}\right) \equiv r^{\alpha} e_{\sigma}^{0}=\left(e_{r}, e_{\theta}\right) \tilde{\boldsymbol{w}}_{0}^{\sigma} .
$$

Substituting this into the homogeneous Lamé equations $((2.1)$ with $f \equiv 0)$ gives

$$
\begin{aligned}
& \tilde{\boldsymbol{L}}\left(\alpha, D_{\theta}\right)\left(\begin{array}{c}
G_{r} \\
G_{\theta}
\end{array}\right) \\
& =\left(\begin{array}{c}
G_{r}^{\prime \prime}+\cot \theta G_{r}^{\prime}+(\hat{v}(\alpha-1)-\alpha-1) G_{\theta}^{\prime}+\hat{v}(\alpha-1)(\alpha+2) G_{r}+(\hat{v}(\alpha-1)-\alpha-1) \cot \theta G_{\theta} \\
\hat{v} G_{\theta}^{\prime \prime}+(\hat{v}(\alpha+2)-\alpha) G_{r}^{\prime}+\hat{v} \cot \theta G_{\theta}^{\prime}+\left(\alpha(\alpha+1)-\hat{v} / \sin ^{2} \theta\right) G_{\theta}
\end{array}\right)=0,
\end{aligned}
$$

where $\hat{v}=2+\lambda / \mu$ and the prime (') denotes differentiation w.r.t. the argument. We have for (2.11) that $L\left(\alpha, D_{\omega}\right)$ $=\left(e_{r} e_{\theta}\right) \tilde{L}\left(\alpha, D_{\theta}\right)$. It is well known that a system of $m$ ordinary differential equations of order $n$ has $m \cdot n$ linearly independent solutions, so for (3.3) there are four such solutions to be found.

We use the Papkovich-Neuber potentials [9] to construct the solutions. Thus

$$
\boldsymbol{u}=4(1-v) \boldsymbol{B}-\nabla\left(\boldsymbol{x} \cdot \boldsymbol{B}+B_{4}\right),
$$

where $v$ is Poisson's ratio $(v=\lambda / 2(\lambda+\mu)), \boldsymbol{B}=\left(B_{1}, B_{2}, B_{3}\right)^{\top}$ and $B_{i}, i=1,2,3,4$, are harmonic functions. In spherical polar coordinates (3.4) becomes

$$
\boldsymbol{u} \equiv\left(\begin{array}{c}
u_{r} \\
u_{\theta} \\
u_{\varphi}
\end{array}\right)=(3-4 v)\left(\begin{array}{c}
\boldsymbol{B} \cdot e_{r} \\
\boldsymbol{B} \cdot e_{\theta} \\
\boldsymbol{B} \cdot e_{\varphi}
\end{array}\right)-\left(\begin{array}{c}
r e_{r} \cdot \frac{\partial \boldsymbol{B}}{\partial r}+\frac{\partial B_{4}}{\partial r} \\
e_{r} \cdot \frac{\partial \boldsymbol{B}}{\partial \theta}+\frac{1}{r} \frac{\partial B_{4}}{\partial \theta} \\
\frac{1}{\sin \theta} e_{r} \cdot \frac{\partial \boldsymbol{B}}{\partial \varphi}+\frac{1}{r \sin \theta} \frac{\partial B_{4}}{\partial \varphi}
\end{array}\right) .
$$

If we now take

$$
B_{1}=B_{2}=0, \quad B_{3}=c_{1} r^{\alpha} F_{\alpha}(\theta), \quad B_{4}=c_{2} r^{\alpha+1} F_{\alpha+1}(\theta),
$$

where, for the $B_{i}$ to be harmonic, $F_{\alpha}(\theta)=P_{\alpha}(\cos \theta)$ or $F_{\alpha}(\theta)=Q_{\alpha}(\cos \theta)$, (the Legendre functions of the first or second kind) then we have, via (3.5), the necessary four linearly independent solutions of (3.3). Of these solutions those arising from $F_{\alpha}(\theta)=Q_{\alpha}(\cos \theta)$ involve unbounded displacements, so we exclude them. Substituting (3.6) with $F_{\alpha}(\theta)=P_{\alpha}(\cos \theta) \equiv P_{\alpha}$ 
into (3.5) we obtain the general form of the rotationally symmetric solutions of the Lamé equations,

$$
\begin{aligned}
\tilde{\boldsymbol{w}}_{0}^{\sigma} & =c_{1} r^{\alpha}\left(\begin{array}{c}
(3-4 v-\alpha) P_{\alpha} \cos \theta \\
P_{\alpha}^{\prime} \cos \theta \sin \theta-(3-4 v) P_{\alpha} \sin \theta
\end{array}\right)+c_{2} r^{\alpha}\left(\begin{array}{c}
-(\alpha+1) P_{\alpha+1} \\
\sin \theta P_{\alpha+1}^{\prime}
\end{array}\right) \\
& \equiv r^{\alpha}\left\{c_{1}\left(\begin{array}{l}
A_{11}(\alpha, \theta) \\
A_{21}(\alpha, \theta)
\end{array}\right)+c_{2}\left(\begin{array}{l}
B_{11}(\alpha, \theta) \\
B_{21}(\alpha, \theta)
\end{array}\right)\right\} \equiv r^{\alpha}\left(c_{1} \tilde{A}+c_{2} \tilde{B}\right),
\end{aligned}
$$

where we have used the notation of (3.1), so that

and

$$
e_{\sigma}^{0}=\left(e_{r} e_{\theta}\right)\left(c_{1} \tilde{A}+c_{2} B\right),
$$

$$
\tilde{A}(\alpha, \theta) \equiv\left(\begin{array}{c}
A_{11}(\alpha, \theta) \\
A_{21}(\alpha, \theta)
\end{array}\right) \equiv\left(\begin{array}{c}
(3-4 v-\alpha) P_{\alpha} \cos \theta \\
\left(P_{\alpha}^{\prime} \cos \theta-(3-4 v) P_{\alpha}\right) \sin \theta
\end{array}\right), \quad \tilde{\boldsymbol{B}}(\alpha, \theta) \equiv\left(\begin{array}{c}
B_{11}(\alpha, \theta) \\
B_{21}(\alpha, \theta)
\end{array}\right) \equiv\left(\begin{array}{c}
-(\alpha+1) P_{\alpha+1} \\
\sin \theta P_{\alpha+1}^{\prime}
\end{array}\right) .
$$

\section{Homogeneous boundary conditions}

We here consider the equations that result from applying the boundary operators $L_{i}, i=1$ or 2 of (2.2) or (2.3) to our solution (3.7). From these we find the values of the $\alpha_{n}$, their multiplicities, $I_{n}$, the eigenvector-functions and associate vector-functions and information about the lengths of the Jordan chains, $J_{a n}$. We define $\left.L_{i}\left(\alpha, D_{\omega}\right) e_{\sigma}^{0}\right|_{\theta=\theta_{0}} \equiv\left(e_{r} e_{\theta}\right) M_{i}\left(\alpha, \theta_{0}\right) c$, $i=1$ or 2 .

For the Dirichlet boundary conditions $(i=1)$ we obtain

$$
M_{1}\left(\alpha, \theta_{0}\right) c \equiv\left(\begin{array}{ll}
A_{11}\left(\alpha, \theta_{0}\right) & B_{11}\left(\alpha, \theta_{0}\right) \\
A_{21}\left(\alpha, \theta_{0}\right) & B_{21}\left(\alpha, \theta_{0}\right)
\end{array}\right)\left(\begin{array}{l}
c_{1} \\
c_{2}
\end{array}\right) .
$$

For the stress-free boundary condition $(i=2)$ we have for this problem that $M(x)=e_{\theta}$ so that $S(\boldsymbol{u}(\boldsymbol{x})) \cdot \boldsymbol{M}(\boldsymbol{x})=\left(\sigma_{\mathrm{r} \theta} \sigma_{\theta \theta} \sigma_{\varphi \theta}\right)^{\top}$ with $\sigma_{\varphi \theta}=0$ due to the rotational symmetry. We obtain

where

$$
M_{2}\left(\alpha, \theta_{0}\right) c \equiv\left(\begin{array}{ll}
A_{12}\left(\alpha, \theta_{0}\right) & B_{12}\left(\alpha, \theta_{0}\right) \\
A_{22}\left(\alpha, \theta_{0}\right) & B_{22}\left(\alpha, \theta_{0}\right)
\end{array}\right)\left(\begin{array}{l}
c_{1} \\
c_{2}
\end{array}\right),
$$

$$
\begin{aligned}
& A_{12}(\alpha, \theta) \equiv\left(P_{\alpha}^{\prime} \cos \theta(\alpha+2 v-2)+P_{\alpha}(2 v-1) \alpha\right) \sin \theta 2 \mu, \\
& A_{22}(\alpha, \theta) \equiv\left(\alpha(\alpha+2 v) P_{\alpha} \cos \theta-\left(1+\sin ^{2} \theta(2 v-3)\right) P_{\alpha}^{\prime}\right) 2 \mu, \\
& B_{12}(\alpha, \theta) \equiv \alpha \sin \theta P_{\alpha+1}^{\prime} 2 \mu, \quad B_{22}(\alpha, \theta) \equiv\left(P_{\alpha+1}(\alpha+1)^{2}-P_{\alpha+1}^{\prime} \cos \theta\right) 2 \mu .
\end{aligned}
$$

For $\alpha$ to be an eigenvalue of our operator $A_{i}\left(\alpha, D_{\omega}\right)$ (see 2.11) with eigenvector-function $\boldsymbol{e}_{\sigma}^{0}$ it is necessary that $\left.L_{i}\left(\alpha, D_{\omega}\right) e_{\sigma}^{0}\right|_{\theta=\theta_{0}}=0$ has a non-trivial solution. From the definition we thus require that

$$
\operatorname{det} M_{i} \equiv \mathrm{D}_{i}\left(\theta_{0}, \alpha\right)=0, \quad i=1 \text { or } 2 .
$$

These equations provide, on simplification, the following transcendental equations from which the eigenvalues, $\alpha_{n}$, are to be evaluated for $\theta_{0} \in(0, \pi)$ :

$$
\begin{aligned}
\mathrm{D}_{1}\left(\theta_{0}, \alpha\right)=\frac{-(\alpha+1)}{\sin \theta_{0}}\left(P_{\alpha}^{2} \cos \theta_{0}(\alpha+4 v-3)\right. & +P_{\alpha} P_{\alpha+1}\left(3-4 v-\cos ^{2} \theta_{0}(2 \alpha+1)\right) \\
& \left.+P_{\alpha+1}^{2} \cos \theta_{0}(\alpha+1)\right)=0, \\
\mathrm{D}_{2}\left(\theta_{0}, \alpha\right)=\frac{2(\alpha+1)^{2}}{\sin ^{3} \theta_{0}} & {\left[P_{\alpha}^{2} \cos \theta_{0}\left(2(1-v) \cos ^{2} \theta_{0}-\alpha(\alpha+1) \sin ^{2} \theta_{0}\right)\right.} \\
& -P_{\alpha} P_{\alpha+1}\left(\alpha(2 \alpha+1) \sin ^{4} \theta_{0}+4(1-v) \cos ^{2} \theta_{0}-\alpha(\alpha+1) \sin ^{2} \theta_{0}\right. \\
& \left.+P_{\alpha+1}^{2} \cos \theta_{0}\left(2(1-v)-\alpha(\alpha+1) \sin ^{2} \theta_{0}\right)\right] \\
=2 \alpha(\alpha+1) & \left(D_{1}(\theta, \alpha)-4(1-v) \sin \theta_{0} P_{\alpha} P_{\alpha}^{\prime}\right)+2(1-v) \sin 2 \theta_{0} P_{\alpha}^{\prime 2}=0 .
\end{aligned}
$$

At each point $\left(\theta_{0}, \alpha_{n}\right)$ where $\mathrm{D}_{i}\left(\theta_{0}, \alpha_{n}\right)=0, i=1$ or 2 , there is a non-trivial solution $c_{i}, i=1$ or 2 , to be found, for which it is necessary to check via (3.8) whether $e_{\sigma}^{0}(r, \omega)$ is an eigenvector-function of the operators $A_{i}\left(\alpha, D_{\omega}\right)$; e.g. $\alpha=-1$ yields no eigenvector-function for the Dirichlet problem. From (4.1) or (4.2) we obtain that $c=\left(B_{1 i}\left(\alpha_{n}, \theta_{0}\right)-A_{1 i}\left(\alpha_{n}, \theta_{0}\right)\right)^{\top}$, $i=1$ or 2 , yields such a solution. Thus, in (3.7), we have that

$$
\tilde{\boldsymbol{w}}_{0, n}^{\sigma}=r^{\alpha_{n}}\left(\tilde{\boldsymbol{A}}\left(\alpha_{n}, \theta\right) B_{1 i}\left(\alpha_{n}, \theta_{0}\right)-\tilde{\boldsymbol{B}}\left(\alpha_{n}, \theta\right) A_{1 i}\left(\alpha_{n}, \theta_{0}\right)\right) \text {. }
$$

We have thus computed the eigenvector-functions $e^{0}\left(\alpha_{n}, \omega\right)$ of (3.8). 


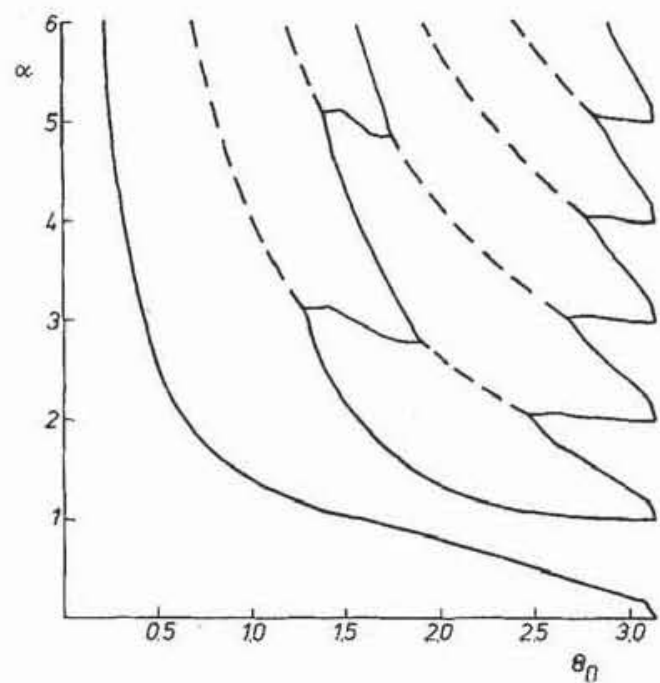

Fig. 2. Values of $\alpha_{n}$, dependence on $\theta_{0}$ for Dirichlet $(i=1)$ boundary condition, $v=0.3$

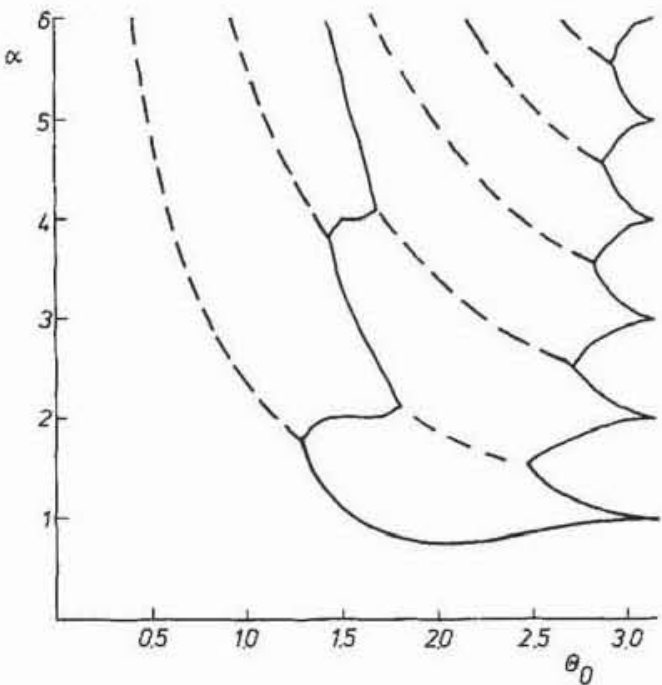

Fig. 3. Values of $\alpha_{n}$, dependence on $\theta_{0}$ for stress-free $(i=2)$ boundary condition, $v=0.3$

We note that the dimension, $I_{n}$, of the eigenspace is unity for $\operatorname{Re}(\alpha)>0$ : For there to be an eigenvector-function that is linearly independent of that above it is necessary in (4.1) or (4.2) that $A_{i j}=B_{i j}=0, j=1,2, i=1$ or 2 . This does not happen for $\theta \in(0, \pi)$. We define $\boldsymbol{w}_{k, n}^{1} \equiv \boldsymbol{w}_{k, n}$ and $\boldsymbol{e}_{1}^{k} \equiv e^{k}$.

When $\alpha=0$, (4.4) is satisfied for all $\theta_{0}$. Substituting $\alpha=0$ in (3.7) we obtain that $\tilde{u}=(\cos \theta-\sin \theta)^{\top}$ so that, in (3.1), $\boldsymbol{u}=\left(\begin{array}{lll}0 & 0 & 1\end{array}\right)^{\top}$. For the stress-free boundary conditions this eigenvector-function is the rotationally symmetric rigid body motion along the $z$-axis. Clearly it is not a singular vector-function. For the Dirichlet boundary conditions there is no non-trivial solution.

We are thus left with equation (4.4) to be solved for $\alpha>0$ given the value of $\theta_{0}$. Solving $\mathrm{D}_{1}\left(\theta_{0}, \alpha\right)=0$ produced the curves of Fig. 2 for $v=0.3$ and solving $\mathrm{D}_{2}\left(\theta_{0}, \alpha\right)=0$ produced those of Fig. 3 also for $v=0.3$. The real values of $\alpha$ are joined by solid lines, whilst the complex values are joined by dashed lines. Our method for obtaining these curves is described in the next section, where we also comment on the values obtained.

We recall formula (2.13) and now investigate the existence of associate vector-functions. The following lemma of NAJMARK [10] gives a connection between the length of the Jordan chains, $J_{n} \equiv J_{1 n}$, and the multiplicity, $m\left(\alpha_{n}\right)$, of the zeros $\alpha_{n}$ of $\mathrm{D}_{i}\left(\theta_{0}, \alpha_{n}\right), i=1$ or 2 .

Lemma:

$$
m\left(\alpha_{n}\right) \geqq J_{n} .
$$

If $m\left(\alpha_{n}\right)=1$ then $\left.\frac{\partial}{\partial \alpha} \mathrm{D}_{i}\left(\theta_{0}\right)\right|_{\alpha=\alpha_{n}} \neq 0$ and there are no associate vector-functions. However at branch points of graphs of $\left(\theta_{n}, \alpha_{n}\right)$ (see Fig. 2 and Fig. 3) the zeros are not simple,

$$
\left.\frac{\partial}{\partial \alpha} \mathrm{D}_{i}\left(\theta_{0}, \alpha\right)\right|_{\alpha=\alpha_{n}}=0
$$

and there are associate vector-functions to be found.

We note that $L\left(\alpha, D_{\omega}\right) e^{0}(\alpha, \omega)=0$ for all $\alpha$ so that

$$
\frac{\partial}{\partial \alpha}\left[L\left(\alpha, D_{\omega}\right)\right] e^{0}(\alpha, \omega)+L\left(\alpha, D_{\omega}\right) \frac{\partial}{\partial \alpha} e^{0}(\alpha, \omega)=0 .
$$

Comparing with (2.12) we see that

if

$$
\left.e^{1}\left(\alpha_{n}, \omega\right) \equiv \frac{\partial}{\partial \alpha} e^{0}(\alpha, \omega)\right|_{\alpha=\alpha_{n}}
$$

$$
\boldsymbol{L}_{i}\left(\left.\frac{\partial}{\partial \alpha} e^{0}(\alpha, \omega)\right|_{\alpha=\alpha_{n}}\right)=0, \quad i=1 \text { or } 2 .
$$

Performing the differentiation we see that (4.9) holds if (4.6) holds, so an associate vector-function, $e^{1}\left(\alpha_{n}, \omega\right)$, is given by (4.8), i.e. from (4.5) and (3.8),

$$
e^{1}\left(\alpha_{n}, \omega\right)=\left.\left(\boldsymbol{e}_{r} \boldsymbol{e}_{\theta}\right)\left(\tilde{A} \frac{\partial}{\partial \alpha} B_{1 i}+B_{1 i} \frac{\partial}{\partial \alpha} \tilde{A}-\tilde{B} \frac{\partial}{\partial \alpha} A_{1 i}-A_{1 i} \frac{\partial}{\partial \alpha} \tilde{B}\right)\right|_{\alpha=\alpha_{n}} .
$$




\section{Algorithmic details}

To find the roots of equations (4.4) we developed the technique described in [11]. The Mehler-Dirichlet formula (see e.g. [12]) gives that

$$
P_{\alpha}(\cos \theta)=\frac{\sqrt{2}}{\pi} \int_{0}^{\theta} \frac{\cos \left(\alpha+\frac{1}{2}\right) t}{\sqrt{\cos t-\cos \theta}} \mathrm{d} t .
$$

The integrand's denominator can be written as

$$
\left[-\sin \left(\frac{t+\theta}{2}\right) \sin \left(\frac{t-\theta}{2}\right)\left(\frac{2}{t-\theta}\right)(t-\theta)\right]^{1 / 2} .
$$

The last factor in (5.2) is passed as a weight to a Gauss-Jacobi integration procedure and the product of the middle two factors is, for $t-\theta<0.001$ approximated using the first two terms in a Taylor series. The NAG [7] integration routine used works adaptively to a specified accuracy and thus $P_{\alpha}(\cos \theta)$ is calculated.

Our strategy for finding $\alpha$ satisfying

$$
F\left(\theta_{0}, \alpha\right)=0
$$

with $F$ given by (4.4) is to initially seek a local minimum of $\left|F\left(\theta_{0}, \alpha\right)\right|$ near a guess $\alpha_{0}$ of $\alpha$ to an accuracy of $10^{-3}$. This is passed, as a first approximation to $\alpha$, to a root finding routine which works on the system formed by the real and imaginary parts of $F$ as functions of the real and imaginary parts of $\alpha$. Use of this strategy was found to ensure convergence to a root. The accuracy to which the $P_{\alpha}\left(\cos \theta_{0}\right)$ were calculated was, to save computational time, restricted by the estimated proximity to the root.

The curves of Fig. 2 and Fig. 3 were obtained by starting from the known, integer, limits as $\theta_{0} \rightarrow \pi$. Each subsequent point was found at a smaller value of $\theta_{0}$ using as initial guesses the previous root plus and minus 0.15 . In this way the branches were located.

We compare these curves with those obtained in [11] for the dominant (smallest) $\alpha, \alpha_{\mathrm{p}}$ for the Poisson problem in the same domain with Dirichlet boundary conditions. In the context of the Lamé equations we denote the dominant $\alpha$ 's for the Dirichlet and stress-free boundary conditions by, respectively, $\alpha_{D}$ and $\alpha_{S}$. We observe that

$$
\text { if }\left\{\begin{array}{c}
0<\theta_{0}<\pi / 2 \\
\theta_{0}=\pi / 2 \\
\pi / 2<\theta_{0}<\pi
\end{array}\right\} \text { then }\left\{\begin{array}{c}
\alpha_{\mathrm{D}}<\alpha_{\mathrm{P}}<\alpha_{\mathrm{S}} \\
\alpha_{\mathrm{D}}=\alpha_{\mathrm{P}}=\alpha_{\mathrm{S}}=1 \\
\alpha_{\mathrm{D}}>\alpha_{\mathrm{P}} \text { and } \alpha_{\mathrm{S}}>\alpha_{\mathrm{P}}
\end{array}\right\} \text {. }
$$

These inequalities were verified for a range of values of $v$, in fact there was seen to be only a small dependence on $v$ for $v$ in the interval $(0,1,0.4)$ (cf. [3]).

The smallest value of $\alpha_{S}, \alpha_{M}$ occurs at an angle $\theta_{0}=\theta_{M}$ depending on $v$. The variation of $\theta_{M}$ (solid line) and $\alpha_{M}$ (dashed line) on $v$ is shown in Fig. 4. It is seen that the minimum value of $\alpha_{M}$ is larger than 0.6681. Using this information and (5.4) we obtain bounds on $\alpha_{\mathrm{S}}$ for any $\theta_{0}$ and $v$.

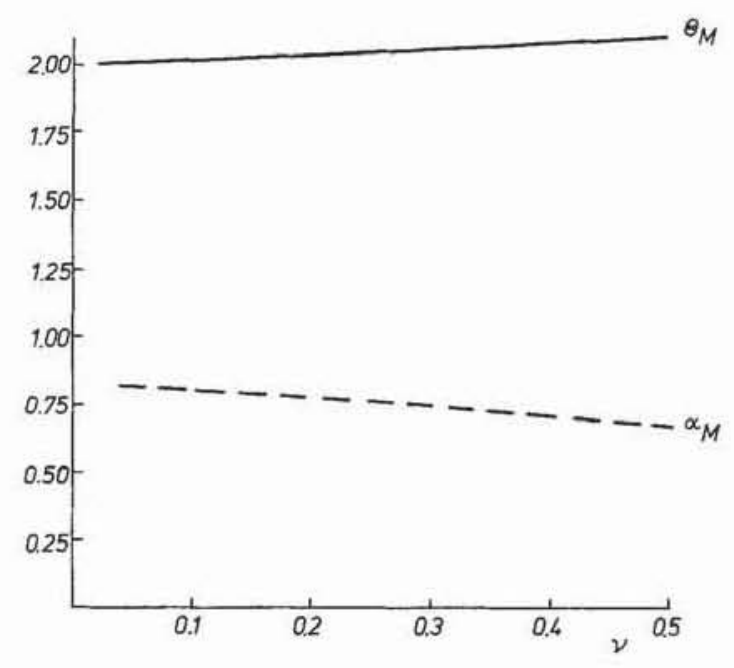

Fig. 4. Variation of $\theta_{\mathrm{M}}$ and $\alpha_{\mathrm{M}}$ with $v$ 


\section{The calculation of the coefficients}

The constants, $C_{\sigma k n}$, in expansion (2.15) for $g_{i}(x) \equiv 0, i=1$ or 2 , can be computed by applying the results of MAZ'JA and Plamenevisij [8]. We consider the cases $m\left(\alpha_{n}\right)=1$ and $m\left(\alpha_{n}\right)=2$ separately.

(i) $m\left(\alpha_{n}\right)=1$ :

$$
C_{10 n} \equiv C_{0 n}=\int_{\Omega} L\left(D_{x}\right)(\eta u) \overline{v_{0 n}} \mathrm{~d} x
$$

where $v_{0 n} \equiv K_{0 n} e^{0}\left(\alpha_{n}^{*}, \omega\right) r^{\alpha_{n}^{*}}, \alpha_{n}^{*}=-\bar{\alpha}_{n}-1, e^{0}$ given via $(4.5)$ and $K_{0 n}$ is a constant which is determined by the orthonormality condition,

$$
\overline{K_{0 n}} \int_{S} \frac{\partial L\left(\alpha_{n}\right)}{\partial \alpha} e^{0}\left(\alpha_{n}, \omega\right) \cdot \overline{e^{0}\left(\alpha_{n}^{*}, \omega\right)} \mathrm{d} \omega \equiv\left(L^{\prime} w_{0}, v_{0}\right)=1
$$

(ii) $m\left(\alpha_{n}\right)=2$ :

$$
C_{10 n} \equiv C_{0 n}=\int_{\Omega} L\left(D_{x}\right)(\eta u) \overline{v_{1 n}} \mathrm{~d} x, \quad C_{11 n} \equiv C_{1 n}=\int_{\Omega} L\left(D_{x}\right)(\eta u) \overline{v_{0 n}} \mathrm{~d} x,
$$

where $v_{1 n} \equiv K_{1 n}\left(\ln r e^{0}\left(\alpha_{n}^{*}, \omega\right)+e^{1}\left(\alpha_{n}^{*}, \omega\right)\right) r^{\alpha_{n}^{*}}$. It is shown in reference [8] that $K_{0 n}$ and $K_{1 n}$ can be chosen to satisfy the following biorthogonality conditions (notation as above),

$$
\begin{aligned}
& \left(L^{\prime} w_{0}, v_{0}\right)=0, \quad\left(L^{\prime} w_{0}, v_{1}\right)+\frac{1}{2}\left(L^{\prime \prime} w_{0}, v_{0}\right)=1, \quad\left(L^{\prime} w_{1}, v_{0}\right)+\frac{1}{2}\left(L^{\prime \prime} w_{0}, v_{0}\right)=1, \\
& \left(L^{\prime} w_{1}, v_{1}\right)+\frac{1}{2}\left(L^{\prime \prime} w_{1}, v_{0}\right)+\frac{1}{2}\left(L^{\prime \prime} w_{0}, v_{1}\right)+\frac{1}{6}\left(L^{\prime \prime \prime} w_{0}, v_{0}\right)=0 .
\end{aligned}
$$

If this is done, the coefficients $C_{0 n}$ and $C_{1 n}$ are given by (6.2) and (6.3), respectively.

\section{Conclusion-theorem}

We have established that rotationally symmetric solutions of boundary value problems for the Lamé equations possess the following expansion near rotationally symmetric vertices.

$$
u(r, \omega)=\sum_{n=1}^{N} \sum_{k=0}^{J_{n}-1} \eta C_{k n} \boldsymbol{w}_{k, n}(r, \omega)+u_{0}(r, \omega):
$$

The conditions are given for expansion (2.15) of Theorem 3. Here the functions $w_{k, n}(r, \omega)\left(\equiv \boldsymbol{w}_{k, n}^{1}(r, \omega)\right.$ of $\left.(2.14)\right)$ involve the values of $\alpha_{n}$, indeed the value of $N$ in (7.1) is similarly determined. The $\alpha_{n}$ are calculated from (4.4) - for $v=0.3$ their values can be read from Fig. 2 or Fig. 3 depending on the relevant boundary conditions. The vector-function $\boldsymbol{w}_{0 n}$ is given by (4.5) using (3.7) or (4.2) (Dirichlet or stress-free boundary conditions). The constant $C_{0 n}$ is given by $(6.1)$ unless $J_{n} \neq 1$. If $J_{n}=2$ (such as at branch points of Fig. 2 or 3 ) then the adjoint vector-function $e^{1}$ of $(4.10)$ is required for $w_{1, n}(r, \omega)$ and the constants $C_{0 n}$ and $C_{1 n}$ are given by (6.2) and (6.3).

Thus we have proved the following

Theorem 4: Let $\boldsymbol{u}$ be a solution from $\left[V^{2+l, p}(\Omega, \beta)\right]^{3}$ of problem (2.1), (2.2) or (2.1), (2.3) for $f \in\left[V^{l^{\prime}, p^{\prime}}\left(\Omega, \beta^{\prime}\right)\right]^{3}$, $g_{1} \in\left[V^{2+l^{\prime}-1 / p^{\prime}, p^{\prime}}\left(\partial \Omega, \beta^{\prime}\right)\right]^{3}$, or $g_{2} \in\left[V^{1+l^{\prime}-1 / p^{\prime}, p^{\prime}}\left(\partial \Omega, \beta^{\prime}\right)\right]^{3}, l^{\prime} \geqq l, h^{\prime}=\beta^{\prime}+3 / p^{\prime}-2-l^{\prime}<\beta+3 / p-2-l=h$. Assume that no eigenvalues of the operators $A_{i}\left(\alpha, D_{\omega}\right), i=1$ or 2 , are situated on the lines $\operatorname{Re}(\alpha)=-h$ and $\operatorname{Re}(\alpha)=-h^{\prime}$. Then the following expansion (see (2.15)) holds near the conical point 0 with angle $\theta_{0} \in(0, \pi)$ :

$$
\begin{aligned}
u(r, \omega)= & \sum_{\substack{n \\
-h<\operatorname{Re}\left(\alpha_{n}\right)<-h^{\prime} \\
m\left(\alpha_{n}\right)=1}} \hat{c}_{0 n^{\prime}} r^{\alpha_{n}} e^{0}\left(\alpha_{n}, \omega\right) \\
& +\sum_{\substack{n \\
-h<\operatorname{Re}\left(\alpha_{n}\right)<-h^{\prime} \\
m\left(\alpha_{n}\right)=2}} c_{0 n^{\prime}} r^{\alpha_{n}} e^{0}\left(\alpha_{n}, \omega\right)+c_{1 n} r^{\alpha_{n}}\left(\ln r e^{0}\left(\alpha_{n}, \omega\right)+e^{1}\left(\alpha_{n}, \omega\right)\right)+u_{0}(r, \omega),
\end{aligned}
$$

where $u_{0}(r, \omega) \in\left[V^{2+l^{\prime}, p^{\prime}}\left(\Omega, \beta^{\prime}\right)\right]^{3}, \hat{c}_{0 n}$ is given by (6.1) (where $\left.c_{0 n} \equiv \hat{c}_{0 n}\right), c_{0 n}$ by $(6.2)$ and $c_{1 n}$ by $(6.3) ; e^{0}\left(\alpha_{n}, \omega\right)$ is computed by (3.8) via (4.5) and $e^{1}\left(\alpha_{n}, \omega\right)$ is given by (4.10); $m\left(\alpha_{n}\right)$ is the multiplicity of the zeros of $D_{i}\left(\theta_{0}, \alpha_{n}\right), i=1$ or $i=2($ see (4.3)).

\section{Acknowledgement}

Part of this work was done whilst Dr. SÄNDIG visited BICOM in 1988 supported by the British Council. The work of Dr. BEAGLES was financed by the SERC under Grant No. GR/D/76745. The support of the Wilhelm-Pieck University Rostock is also acknowledged. 


\section{References}

1 KondRAT'Ev, V. A.: Boundary value problems for elliptic equations in domains with conical or angular points. Trudy Moskovkogo Mat. Obschetsva, 16 (1967), 209-292, and Trans. Moscow Math. Soc. 16 (1967), 227-301.

2 Maz'JA, V. G.; Plamenevskis, V. A.: The first boundary value problem for classical equations of Mathematical Physics in domains with piecewise smooth boundaries. ZAA 2 (1983) 4, 335-359.

3 BAŽANT, Z. P.; KeER, L. M.: Singularities of elastic stresses and of harmonic functions at conical notches or inclusions. Internat. J. Solids Structures 10 (1974), 957-964.

4 Grisvard, P.: Elliptic problems in non-smooth domains. Pitman 1985.

5 Grisvard, P.: Problèmes aux limites dans les polygônes. Mode d'emploi EDF Bulletin, Sér. C (1986) No. 1, 21-59.

6 MAZ'JA, V. G.; PlamenevskiJ, V. A.: Estimates in Lp and in Hölder classes and the Miranda-Agmon maximum principle for solutions of elliptic boundary value problems in domains with singular points on the boundary. Math. Nachr. 81 (1978), 25-82, and Amer. Math. Soc. Transl. 123 (1984) (2), 1-56.

7 KUfNER, A.; SÄNDIG, A.-M.: Some applications of weighted Sobolev spaces. Teubner Texte 1987.

8 Maz'Ja, V. G.; PlamenevskiJ, V. A.: About the coefficients in the asymptotics of the solutions of elliptic boundary value problems in domains with conical points. Math. Nachr. 76 (1977), 29-60.

9 Atkin, R. J.; Fox, N.: An introductions to the theory of elasticity. Longman Mathematical Texts 1980.

10 NAJMARK, M. A.: Linear differential operators. Nauka 1969.

11 Beagles, A. E.; Whiteman, J. R.: General conical singularities in three-dimensional Poisson problems. Math. Meth. Appl. Sci. 11 (1989), 215-228.

12 Erdelyi, A.; Magnus, W.; OBerhettinger, F.; Tricomi, F. G.: Higher transcendental functions. Vol. 1. Bateman Manuscript Project, California Institute of Technology, McGraw-Hill 1953

13 NAG, Numerical Algorithms Group. Oxford 1984.

Received September 21, 1989

Addresses: Doz. Dr. s.c. A.-M. SÄNDIG, Wilhelm-Pieck-Universität Rostock, Sektion Mathematik, Universitätsplatz 1, O-2500 Rostock, Germany;

Dr. A. E. Beagles, Brunel University, Department of Mathematics and Statistics, Uxbridge, Middlesex UB8 3PH, U.K. 